\title{
THE EFFECT OF ELECTRODE CONFIGURATION ON SPECTRAL DISTANCE MEASURES
}

\author{
Wen-Juh Kang ${ }^{1}$, Cheng-Kung Cheng ${ }^{2}$, Jin-Shin Lai ${ }^{3}$, \\ Hen-Wei Tsao ${ }^{1}$ and Te-Son Kuo ${ }^{1}$ \\ ${ }^{1}$ Department of Electrical Engineering, ${ }^{2}$ Center for Biomedical Engineering and \\ ${ }^{3}$ Department of Rehabilitation, National Taiwan University, R.O.C.
}

\begin{abstract}
We measure EMG signals of different moitons with two electrode configurations simultaneously. Electrode pairs are located separately on dominant muscles (S-type) and closely in the region between muscles (C-type). The application of cepstral method in C-type signal doesn't achieve the same improvement as in S-type. The bandwidth of signal and the amplified noise in high-frequency band (HFB) are supposed to be responsible for the above degradation. This study provides theoretical verification for the above explanation.
\end{abstract}

\section{Introduction}

As for motions with two dominant muscles, we have tried to place surface electrodes directly on the active muscles to minimize the loss of information. It has been shown that cepstral method achieves remarkable improve-ment over conventional AR method in the discrimination of S-type EMG signals [1]. In this study, we measure S-type and C-type signals at the same time. The recorded signals are then analyzed with $A R$ and cepstral methods. The purpose is to investigate the performance of these two spectral distance measures in different electrode configurations.

\section{Experiments}

Four normal volunteers participated in this study. Electrodes were placed on and between the sternocleidomastoid and the upper trapezius bilaterally. Each subject was instructed to perform ten motions of head and shoulder as described in the previous investigation[1]. The selected motions were designed for quadriplegia. EMG signals of both types of electrode configuration were recorded and analyzed.

The relation between S-type and C-type signals

C-type signal is obtained from S-type signal through the tissue and a differential pair of electrodes which can be deemed as a lowpass filter and a highpass filter, respectively. As a result, the bandwidth of C-type signal is narrower than that of S-type. The noise added during the process of transmission is greatly amplified in the HFB of C-type signal.

Spectral meanings for Euclidean distances of $A R$ and cepstral parameters

It is assumed that EMG signals can be modeled as a pure AR process. The normalized power spectral density is given by $1 /|A(\xi)|^{2}$ where

$A(\xi)=1+\sum_{i=1}^{P} a(i) e^{-j 2 \pi \xi}$

a(i) being the AR parameters and $P$ being the model's order. The Euclidean distance between AR parameters of reference signal and test signal is

$d_{A R}^{2}=\sum_{i=1}^{p}\left[a_{i}(i)-a_{r}(i)\right]^{2}=\int_{0}^{1}\left|A_{i}(\xi)-A_{r}(\xi)\right|^{2} d \xi$

where the subscripts $t, r$ denote the test set and the reference set, respectively.

The cepstral distance is given by

$d_{C E P}^{2}=\sum_{i=1}^{p}\left[c_{t}(i)-c_{r}(i)\right]^{2}=\int_{0}^{1}\left|\ln A_{t}(\xi)-\ln A_{r}(\xi)\right|^{2} d \xi$

In general, $d_{A R}$ is dominated by the spectral difference(SPD) in HFB, while the SPD is more equally weighted in $d_{\text {CEP. }}$. The plots of power spectral density show that the main SPD of various motions appears in lowfrequency band (LFB). Therefore, the performance of cepstral method is usually superior to AR method. 
The effect of bandwidth on the performance of cepstral method

Let $A(\xi)$ be simplified as

$$
\begin{aligned}
& A(\xi)=q_{L} \text { for } 0 \leq \xi<B \\
& =q_{H} \quad \text { for } \quad B \leq \xi<1
\end{aligned}
$$

where $q_{H}$ is in general 3 or 4 orders of magnitude larger than $q_{L}, B$ being the bandwidth of signal. Then it can be proved that

$d_{A R}^{2}=\left|q_{r L}-q_{t L}\right|^{2} B+\left|q_{r H}-q_{t H}\right|^{2}(1-B)=E_{A L}+E_{A H}$ $d_{C E P}^{2}=\left|\ln \left(\frac{q_{r L}}{q_{t L}}\right)\right|^{2} B+\left|\ln \left(\frac{q_{r H}}{q_{t H}}\right)\right|^{2}(1-B)=E_{C L}+E_{C H}$

where $E$ being the SPD, the subscripts $L$ and $H$ denote LFB and HFB, $A$ and $C$ represent AR and cepstral methods, respectively. Table I shows the simulation results for different value of $B$. When the SPD in LFB is greater than that in HFB, the distance is dominated by the SPD in LFB which contains most discriminative information. When $B$ varies from 0.6 to 0.4 , the relative importance between $E_{C L}$ and $E_{C H}$ exchanges. Therefore, cepstral method is more sensitive to the variation of bandwidth. Narrower bandwidth results in worse performance for cepstral method.

TABLE I

SIMULATION RESULTS FOR THE EFFECT OF BANDWIDTH

\begin{tabular}{|l|l|l|l|l|}
\hline $\mathrm{B}$ & $\mathrm{E}_{\mathrm{AL}}$ & $\mathrm{E}_{\mathrm{AH}}$ & $\mathrm{E}_{\mathrm{CL}}$ & $\mathrm{E}_{\mathrm{CH}}$ \\
\hline 0.4 & 0.0001 & 15 & 0.192 & 0.288 \\
\hline 0.5 & 0.000125 & 12.5 & 0.24 & 0.24 \\
\hline 0.6 & 0.00015 & 10 & 0.288 & 0.192 \\
\hline \multicolumn{5}{|c}{$\mathrm{q}_{\mathrm{rL}}=0.05 \quad \mathrm{q}_{\mathrm{tL}}=0.1 \quad \mathrm{q}_{\mathrm{rH}}=5 \quad \mathrm{q}_{t H}=10$}
\end{tabular}

The influence of noise on the performance of AR method

Suppose that $n_{H}$ is the amplified noise in HF band, it can be shown that

$$
\begin{aligned}
A_{n}(\xi) & =q_{L} \quad \text { for } 0 \leq \xi<B \\
& =q_{H} / 1+q_{H} n_{H} \quad \text { for } B \leq \xi<1
\end{aligned}
$$

\begin{tabular}{|c|c|c|}
\hline $\mathrm{n}_{\mathrm{H}}$ & $\mathrm{E}_{\text {AH }}$ & $E_{C H}$ \\
\hline 0.0 & 0.5 & 0.0249 \\
\hline 0.2 & 0.0386 & 0.0069 \\
\hline 0.4 & 0.0082 & 0.0032 \\
\hline 0.6 & 0.0027 & 0.0018 \\
\hline
\end{tabular}

TABLE II SIMULATION RESULTS FOR THE EFFECT OF NOISE

Table II shows the simulation results for various levels of noises. It is obvious that the ratio of the SPD in LFB to that in HFB varies much faster for AR method. As noise level raises, $E_{A L}$ becomes more important in the calculation of distance. Consequently, the performance of AR method is promoted by the noise in HFB.

The recognition rates for different electrode configurations are shown in Table III. As a result of the mentioned reasons, the performance of cepstral method degrades for C-type signals.

TABLE III

RECOGNITION RATES FOR TWO ELECTRODE CONFIGURATIONS

\begin{tabular}{|l|l|l|l|l|}
\multicolumn{2}{c}{} & \multicolumn{2}{c}{ C-TYPE(\%) } & \multicolumn{2}{c|}{ S-TYPE(\%) } \\
\hline SUBJECT & AR & CEP & AR & CEP \\
\hline$\# 1$ & 69.5 & 73.5 & 79.5 & 87.0 \\
\hline$\# 2$ & 66.0 & 67.0 & 68.5 & 82.0 \\
\hline$\# 3$ & 75.5 & 75.0 & 72.0 & 76.5 \\
\hline$\# 4$ & 76.0 & 75.0 & 75.0 & 84.0 \\
\hline
\end{tabular}

\section{Conclusions}

The combination of cepstral method and Stype electrode configuration is recommended for the discrimination of motions with two dominant muscles.

\section{Reference}

[1] W.J. Kang et al., "Cepstral Coefficients as the New Features for EMG Pattern Recognition", Proc. IEEE EMBS San Diego, pp.11431144, 1993. 